\title{
Can a supranational medicines agency restore trust after vaccine suspensions? The case of Vaxzevria
}

\section{Andrea Albanese ( $\square$ andrea.albanese@liser.lu )}

Luxembourg Institute of Socio-Economic Research

Francesco Fallucchi

University of Parma

\section{Bertrand Verheyden}

Luxembourg Institute of Socio-Economic Research

\section{Research Article}

Keywords: COVID-19, vaccine hesitancy, supranational regulation, public health, regression discontinuity design

Posted Date: January 31st, 2022

DOI: https://doi.org/10.21203/rs.3.rs-1250919/v1

License: (c) (1) This work is licensed under a Creative Commons Attribution 4.0 International License. Read Full License 


\title{
Can a supranational medicines agency restore trust after vaccine suspensions? The case of Vaxzevria
}

\author{
Andrea Albanese ${ }^{1}$, Francesco Fallucchi ${ }^{2} \&$ Bertrand Verheyden $^{1}$ \\ ${ }^{1}$ Luxembourg Institute of Socio-Economic Research (LISER) \\ ${ }^{2}$ University of Parma
}

\begin{abstract}
Over the first half of March 2021, the majority of European governments suspended Astrazeneca's Vaxzevria vaccine as a precaution following media reports of rare blood clots. We analyse the impact of the European Medicines Agency's (EMA) March 18th statement assuring the public of the safety of Vaxzevria and the immediate reinstatement of the vaccine by most countries on respondents' intention to get vaccinated. By relying on survey data collected in Luxembourg and neighbouring areas between early March and mid-April, we observe that the willingness to be vaccinated was severely declining in the days preceding the EMA statement. We implement a regression discontinuity design exploiting the time at which respondents completed the survey and find that the vaccine reinstatement substantially restored vaccination intentions.
\end{abstract}

Keywords: COVID-19, vaccine hesitancy, supranational regulation, public health, regression discontinuity design

JEL classification codes: I12, I18, C21, H12, H40

Corresponding author: andrea.albanese@ liser.lu - Maison des Sciences Humaines 11, Porte des Sciences, 4366 Esch-sur-Alzette, Luxembourg. The authors contributed equally to the paper. 


\section{Introduction}

In 2019, the World Health Organization (WHO) had declared vaccine hesitancy as one of the ten main threats to global health. One year later, public authorities around the world welcomed vaccines as an effective solution to alleviate pressures on health systems and on the economy. Despite months of lockdown and millions of deaths, vaccine hesitancy and antivax movements immediately challenged the vaccination campaign [1]. Concerns related to vaccines were further fueled in early March 2021, when European media reported rare cases of blood clots among people who received Astrazeneca's Vaxzevria vaccine. This led European governments to implement a large and uncoordinated wave of precautionary suspensions. By March 15th, 18 countries had suspended VaxzevriaOn March 18th, the EMA made gave a press conference stating that "the vaccine's proven efficacy in preventing hospitalization and death from COVID-19 outweighs the extremely small likelihood of developing" blood clots, and recommended the vaccine's use. Within a few hours, 15 governments reintroduced Vaxzevria.

This case study provides a natural experiment to assess the impact of a supranational health agency's communication on vaccine hesitancy. In this paper, we use a regression discontinuity design (RDD) to measure the impact of the EMA's statement. We use survey data on the attitudes towards COVID-19 measures of residents of Luxembourg and neighboring regions of Belgium, France and Germany between early March and mid-April 2021. Our RDD approach uses the time of the EMA press conference as cut-off (March 18th at 17:00 CET) and exploits the information of the time of survey responsesWe find an immediate and statistically significant effect on respondents' vaccination intentions, which results from the combination of the EMA's declaration and of the ensuing coordinated reinstatement of the vaccine by the EU member states. Our results are robust to validation tests, density tests and RDD tests on covariates.

Most papers assessing the effectiveness of public interventions against vaccine hesitancy are generally focused on policies which target specific populations and involve interpersonal interactions. [2] provide a meta analysis of 33 studies showing that community-based interventions (generally targeting parents or caregivers of children via home visits or information campaigns through community health workers, as well as reminder interventions), monetary incentives aimed at alleviating financial constraints [3], and technology-based health literacy have significant effects. However, less is known about the effectiveness of large scale official communications about vaccine safety. Our paper contributes to this scarce literature, which was essentially concentrated on the case of the joint vaccine against measles, mumps and rubella (MMR). False concerns of a possible link between MMR vaccination and autism indeed led to a decline in that vaccine -as well as in other vaccines- uptake in many countries. The misinformation's overturn, first by researchers and then by public authorities, allowed to raise vaccine uptake [4-6].

We first present the descriptive statistics of our sample, followed by OLS estimations on the individual determinants of the willingness to be vaccinated. We then present the RDD estimates on the effect of the EMA statement, and conclude. 


\section{Empirical analysis}

\subsection{Data and descriptive statistics}

Our cross-sectional data come from an online survey conducted among the residents of Luxembourg and the border regions. The survey was organized by Luxembourg Institute of SocioEconomic Research (LISER) in collaboration with the University of Luxembourg and advertised at the beginning of March on social media and on some local council websites. After a general section on demographic characteristics respondents were redirected to one of four randomly assigned blocks of questions covering various themes. Our block of interest, which 696 individuals completed, concerns attitudes towards COVID-19 measures.

Table 3 in the Online Appendix shows the summary statistics of the survey respondents. Over the survey period (beginning of March to mid-April 2021), 83\% of respondents claimed that they were willing to get vaccinated. These respondents were mostly women (67\%), with a representative proportion of adults employed (78\%) and with some tertiary education (55\%). The sample is composed of $59 \%$ of individuals who have the Luxembourg nationality. The median household income interval is between $€ 6,000$ and $€ 8,000$, and $37 \%$ of respondents' have a household income strictly above it. COVID-19 is considered dangerous by $68 \%$ of our sample, which contains $44 \%$ of individuals above the age of 50 . Almost two thirds of the sample watch television at least once per day to get informed about the news. Finally, $64 \%$ of respondents have a strong confidence in the Luxembourgish government's action, whereas $36 \%$ either have limited trust or no trust, or did not want to express an opinion. Following the same classification, $48 \%$ did not express strong confidence in the scientific community.

\subsection{Determinants of the willingness to be vaccinated}

A vast literature analyses the determinants of the willingness to be vaccinated. Vaccine hesitancy is linked to low education and income [7], to minority ethnic groups [8], to the use of specific information channels $[9,10]$ as well as to personality traits [11, 12].Table 1 in the Online Appendix provides estimates of a linear probability model with incremental sets of covariates. Results from these regressions are in line with previous research on the determinants of vaccination propensity, as the willingness to be vaccinated does not significantly differ between women and men, or between single and married respondents. Respondents who attained higher education are significantly more willing to be vaccinated than others in all specifications. We also find a weak positive effect among employed individuals and among respondents above 50 years of age, whereas having a household income above the median plays no role on vaccination intentions. Respondents who consider COVID-19 to be dangerous given their age have a significantly stronger intention to be vaccinated, by about 20 percentage points. Getting informed through television at least once per day is associated with a higher propensity to get vaccinated. Finally, respondents who do not express a strong degree of trust in the government's action, and/or in the scientific community, are also less willing to be vaccinated. 
Table 1: Determinants of the willingness to be vaccinated: linear regressions

\begin{tabular}{|c|c|c|c|}
\hline & $(1)$ & (2) & (3) \\
\hline Woman & $\begin{array}{l}0.039 \\
(1.26)\end{array}$ & $\begin{array}{c}0.0133 \\
(0.46)\end{array}$ & $\begin{array}{l}0.015 \\
(0.53)\end{array}$ \\
\hline Luxembourg national & $\begin{array}{c}0.053^{*} \\
(1.76)\end{array}$ & $\begin{array}{l}0.045 \\
(1.58)\end{array}$ & $\begin{array}{l}0.042 \\
(1.55)\end{array}$ \\
\hline Single & $\begin{array}{l}0.040 \\
(0.96)\end{array}$ & $\begin{array}{l}0.052 \\
(1.32)\end{array}$ & $\begin{array}{l}0.052 \\
(1.35)\end{array}$ \\
\hline Graduate & $\begin{array}{c}0.127^{* * *} \\
(4.27)\end{array}$ & $\begin{array}{c}0.129^{* * *} \\
(4.50)\end{array}$ & $\begin{array}{c}0.089^{* * *} \\
(3.18)\end{array}$ \\
\hline Employed & $\begin{array}{l}0.060 \\
(1.63)\end{array}$ & $\begin{array}{c}0.074^{* *} \\
(2.14)\end{array}$ & $\begin{array}{c}0.075^{* *} \\
(2.26)\end{array}$ \\
\hline Age $\geq 50$ & $\begin{array}{c}0.123^{* * *} \\
(3.99)\end{array}$ & $\begin{array}{c}0.060^{* *} \\
(1.99)\end{array}$ & $\begin{array}{c}0.066^{* *} \\
(2.30)\end{array}$ \\
\hline Considers COVID-19 to be dangerous & & $\begin{array}{c}0.244^{* * *} \\
(8.28)\end{array}$ & $\begin{array}{c}0.202^{* * *} \\
(7.04)\end{array}$ \\
\hline Household income above the median & & $\begin{array}{l}0.036 \\
(1.23)\end{array}$ & $\begin{array}{l}0.022 \\
(0.80)\end{array}$ \\
\hline Daily TV information & & $\begin{array}{c}0.108^{* * *} \\
(3.73)\end{array}$ & $\begin{array}{c}0.108^{* * *} \\
(3.89)\end{array}$ \\
\hline Lack of trust in government & & & $\begin{array}{c}-0.123^{* * *} \\
(-4.22)\end{array}$ \\
\hline Lack of trust in science & & & $\begin{array}{c}-0.134^{* * *} \\
(-4.77)\end{array}$ \\
\hline Constant & $\begin{array}{c}0.597^{* * *} \\
(11.24)\end{array}$ & $\begin{array}{c}0.387^{* * *} \\
(7.06)\end{array}$ & $\begin{array}{c}0.548^{* * *} \\
(9.73)\end{array}$ \\
\hline $\mathrm{R}^{2}$ & 0.048 & 0.166 & 0.237 \\
\hline $\mathrm{N}$ & 673 & 673 & 673 \\
\hline
\end{tabular}

$t$ statistics in parentheses

${ }^{*} p<0.1,{ }^{* *} p<0.05,{ }^{* * *} p<0.01$ 


\subsection{Regression discontinuity design}

On March 18th the EMA held a press conference to provide assurance about the safety of the Vaxvervria vaccine. We consider this statement as our treatment. Our identification strategy is based on the comparison of the intentions to be vaccinated between individuals who responded shortly before and shortly after the announcement, on March 18th at 17:00. This comparison is based on the assumption that the composition of both groups of respondents is similar at the cutoff. We therefore implement an RDD estimator using the time of response as the running variable. We follow the standard approach of the literature, running a local linear regression using the optimal mean squared error criterion for each side of the cutoff and triangular kernel weights [13].

The RDD estimator is obtained by running a local linear regression using the optimal mean squared error criterion for each side of the cutoff and triangular kernel weights [13]. This regression is based on the following linear model:

$$
\begin{aligned}
y_{i} & =\alpha+\delta \cdot \mathbb{1}\left(z_{i} \geq 17: 00,18 / 03 / 21\right)+\beta z_{i} \cdot \mathbb{1}\left(z_{i}<17: 00,18 / 03 / 21\right) \\
& +\gamma z_{i} \cdot \mathbb{1}\left(z_{i} \geq 17: 00,18 / 03 / 21\right)+\varepsilon_{i},
\end{aligned}
$$

where

- $y_{i}$ is the outcome variable, which is equal to 1 if respondent $i$ intends to get vaccinated and zero otherwise;

- $\alpha$ is the constant term;

- $z_{i}$ is the exact time at which respondent $i$ finished the survey;

- $\mathbb{1}(\cdot)$ is the indicator function, which is equal to 1 if the argument is true. Therefore, $\mathbb{1}\left(z_{i} \geq 17: 00,18 / 03 / 21\right)$ is a dummy indicator equal to 1 if respondent $i$ completed the survey after the EMA statement.

- $\delta$ is the causal effect of the EMA statement on the outcome;

- $\beta z_{i} \cdot \mathbb{1}\left(z_{i}<17: 00,18 / 03 / 21\right)$ is the linear spline on the left of the cutoff.

- $\gamma z_{i} \cdot \mathbb{1}\left(z_{i} \geq 17: 00,18 / 03 / 21\right)$ is the linear spline on the right of the cutoff;

- $\varepsilon_{i}$ is the idiosyncratic error term (with zero conditional mean).

We implement two extensions of this baseline model. First, we add a set of control variables $X$, which include the determinants used in Section 2.2 as well as the time of responses (morning, afternoon, evening/night) and the day of the week (weekday or weekend). Time-of-response variables are aimed at controlling for changes in the composition of respondents over specific moments of the day and week. Second, we allow for a local quadratic spline.

In Figure 1 and Table 2, we report the RDD estimates of the effect of the EMA statement of the 18th of March. Column (1) shows the estimates from the local linear regression of equation (1) without covariates. Column (3) provides linear regression results controlling for the covariates used in the preliminary analysis and the time-of-response variables. Columns (2) and (4) follow the same logic (without and with covariates) but using a more flexible (local quadratic) spline. Figure 1 provides a graphical representation of the results obtained in columns (3) and (4), i.e. 
Figure 1: The impact of the EMA statement on the willingness to get vaccinated.

(a) Local linear regression

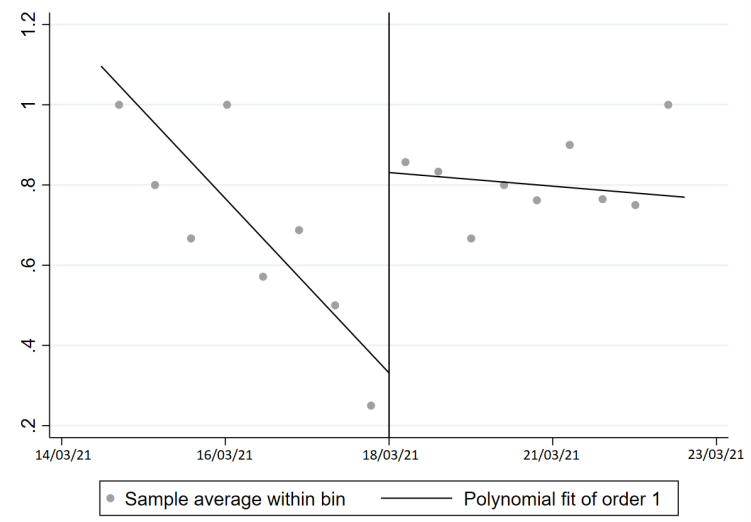

(b) Global-quartic polynomial

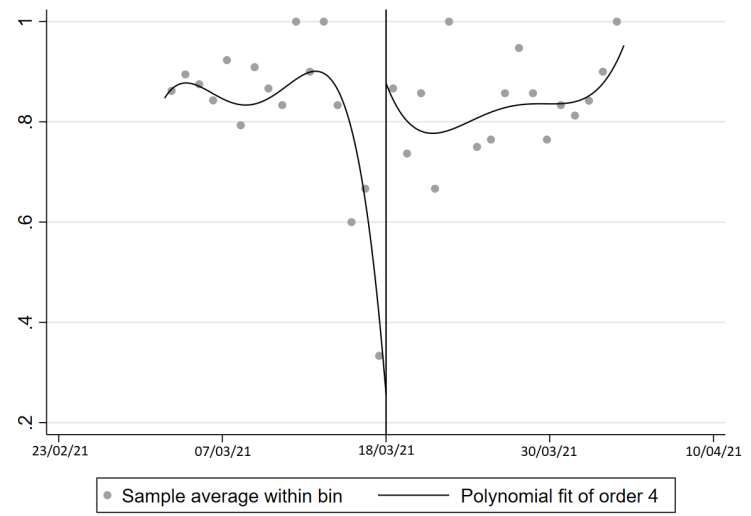

Notes: These graphs show RDD plots for the dependent binary variable equal to 1 if the individual is willing to get vaccinated. Graph (a) is obtained using a local linear polynomial regression with triangular weights and bandwidth following the optimal mean squared error criterion in [13]. Graph (b) is obtained using a global -quartic polynomial and uniform weights.

local linear regression and spline with controls. We find a substantial positive effect of the EMA statement on the intention to be vaccinated of almost 50 percentage points. This effect is statistically significant at the $1 \%$ level and robust to different specifications. Considering the overall evolution of the outcome, the EMA statement was able to sharply invert the collapsing confidence observed in our data. This decline in intentions to get vaccinated in the pre-EMA period is in line with Google Trends data, which shows an increase in searches for "blood clot" and "Astrazeneca" in the days preceding the EMA's statement across the "Greater Region", i.e. Luxembourg, France, Germany and Belgium (see Figure 2 in the Online Appendix).

We further our analysis by conducting several validation tests on the identifying assumptions. First, we implement standard density tests proposed in the literature [13] to check for the existence of mass points on the number of respondents around the cutoff, which may be a sign of manipulation. The density test of [14] confirms that this is not the case, with a robust bias-corrected p-value of 0.962 (see Figure 3). Second, we show that the observable composition of the respondents does not change at the cutoff by obtaining RDD estimates for our set of covariates $X$ (see Table 4). Third, we implement a battery of placebo tests for each side by imposing a false cutoff on each day at 17:00. As shown in Figure 4, the estimates are never statistically significant. Fourth, we test the robustness of our results to the definition of our running variable $z$ by using the time at which respondents started the survey. Table 5 in the Appendix shows that this definition of the running variable delivers even larger point estimates, though still within the confidence interval of our benchmark results. 
Table 2: RDD estimates

(1)

Effect at cut-off
Robust $p$-value
Robust $95 \%$ CI
Y right
Y left
BW Loc. Poly. [h] - left
BW Loc. Poly. [h] - right
BW Bias [b] - left
BW Bias [b] - right
Order loc. poly. [p]
Order bias [q]
Covariates
N

\section{1}

2

No

c
(2)

$0.484^{* * *}$

0.007

[0.143;0.926]

0.827

0.343

14/03/21 02:42

24/03/21 20:37

10/03/21 20:53

30/03/21 13:29

Eff. $\mathrm{N}$ estimate [h]

Eff. $\mathrm{N}$ bias [b]
$0.518 * * *$

0.004

[0.187;0.969]

0.829

0.311

10/03/21 01:03

$31 / 03 / 2116: 23$

07/03/21 22:28

04/04/21 17:42
(3)

$0.488 * * *$

0.001

[0.213;0.828]

0.816

0.325

14/03/21 06:46

24/03/21 03:59

11/03/21 09:42

29/03/21 04:53
(4)

$0.476 * * *$

0.008

[0.133;0.879]

0.831

0.219

Notes: The dependent binary variable is equal to 1 if the individual responds that they are willing to be vaccinated. $z$ is the running variable on the time of survey completion, with a cut-off on the 18th of March at 17:00. We follow [13] with the following options: triangular kernel; variance-covariance matrix estimated using the heteroskedasticity-robust nearest-neighbour variance estimator. Different models: (1) local linear polynomial regression based on the MSE-optimal bandwidth selector for each side of the cut-off, (2) local quadratic polynomial, (3) local linear polynomial regression adding covariates, (4) local quadratic polynomial adding covariates. The table shows the optimal bandwidth for each side of the cut-off for the estimate (h) and the bias (b). * significant at the $10 \%$ level, $* *$ significant at the $5 \%$ level, $* * *$ significant at the $1 \%$ level.

Finally, we create two alternative dependent variables. The information about the willingness to be vaccinated was collected via the following question: Do you intend to get vaccinated against COVID-19? Yes, absolutely (1) - Probably yes (2) - Probably not (3) - No (4). From this question our main outcome variable was based on a dummy variable taking a value of 1 if respondents answered (1) or (2), and a value of 0 otherwise. We therefore check the sensitivity of our results to different versions of the dependent variable by either opposing the definite 'No' (4) to the other answers, or the definite 'Yes' (1) to the other answers. We show in Table 6 in the Appendix that the results are robust to the first alternative formulation of the dependent variable, suggesting that the reinstatement led some individuals who definitely did not want to be vaccinated to reconsider their refusal. In contrast, opposing the 'Yes, absolutely' (1) to all other possible responses does not yield conclusive results (see Table 7). This is sensible since it is less likely that individuals who were already mildly in favour of vaccination would become absolutely convinced by the reinstatement.

To conclude, our validation and robustness tests confirm the reliability of our estimates in representing the causal effect of the EMA statement of the 18th of March. 


\section{Discussion}

The global vaccination campaign against COVID-19 is one of the most crucial challenges in recent history, and vaccine hesitancy is arguably the most important factor threatening its success. The vaccination rates in the most proactive countries are indeed struggling to reach sufficient levels for the acquisition of herd immunity (see Figure 5). Various attempts are being made to convince vaccine-hesitant individuals to reconsider their position through 'carrot and stick' policies. The emergence of more aggressive variants makes the battle against vaccine hesitancy all the more pressing.

Most of the research on public policies aiming to address vaccine hesitancy has focused on targeted interventions [2]. Recently in Europe, public actions fuelled hesitancy when 18 European governments suspended Vaxvevria following news reports of rare blood clots. Our data confirm that in the days that followed these events, intentions to get vaccinated severely declined.

In this paper, we study whether the communication of a supranational drug regulator, the EMA, and the coordinated vaccine reinstatement by 15 governments could restore intentions to get vaccinated to previous levels. While the intention to get vaccinated was at its lowest in the days preceding the EMA statement, we find that the vaccine's reinstatement led to an increase in the intention to get vaccinated of about 50 percentage points.

Interestingly, our finding that the endorsement of the official regulator followed by a coordinated action allowed to rebuild confidence had been hypothesized by [15]. Considering the level of advancement of the vaccination phase, in which significant efforts and investments have been made, this result is particularly important. Moreover, this result has wider implications as the lack of coordination observed at the European level is susceptible to occurring at more local levels of governance. Our results establish that maintaining a common vision and centralized approach is essential to reaching herd immunity at a global level.

Regarding the determinants of vaccination intentwe find that the willingness to get vaccinated is lower among people with a lower educational attainment, among individuals below the age of 50, and among individuals who do not perceive COVID-19 to be dangerous. Lack of trust in science and in government action also correlate negatively with the willingness to get vaccinated, whereas frequent traditional media consumption (TV) is positively correlated with vaccination intent.

Finally, we acknowledge that a reported intention to get vaccinated may differ from individuals' actual behaviour. Still, our results capture a clear break between the pre- and post-EMA declaration periods. In particular, the spectacular drop in the propensity to vaccinate observed in the days preceding the EMA statement could only be triggered by a change in the perceived safety of the vaccines. The vaccine's reinstatement seems to have flattened this wave of panic. 


\section{References}

1. Williams L, Flowers P, McLeod J, Young D, Rollins L, et al. Social patterning and stability of intention to accept a COVID-19 vaccine in Scotland: Will those most at risk accept a vaccine? Vaccines 2021;9:17.

2. Singh P, Dhalaria P, Ghosh S, et al. Strategies to Overcome Vaccine Hesitancy: A Systematic Review. 2020.

3. Banerjee AV, Duflo E, Glennerster R, and Kothari D. Improving immunisation coverage in rural India: clustered randomised controlled evaluation of immunisation campaigns with and without incentives. BMJ 2010;340.

4. Anderberg D, Chevalier A, and Wadsworth J. Anatomy of a health scare: Education, income and the MMR controversy in the UK. Journal of Health Economics 2011;30.

5. Chang LV. Information, education, and health behaviors: Evidence from the MMR vaccine autism controversy. Health Economics (United Kingdom) 2018;27.

6. Carrieri V, Madio L, and Principe F. Vaccine hesitancy and (fake) news: Quasi-experimental evidence from Italy. Health Economics (United Kingdom) 2019;28:1377-82.

7. Paul E, Steptoe A, and Fancourt D. Attitudes towards vaccines and intention to vaccinate against COVID-19: Implications for public health communications. The Lancet Regional Health - Europe 2021;1.

8. Razai MS, Osama T, McKechnie DGJ, and Majeed A. COVID-19 vaccine hesitancy among ethnic minority groups. BMJ 2021;372.

9. Goldstein S, MacDonald NE, Guirguis S, et al. Health communication and vaccine hesitancy. Vaccine 2015;33:4212-4.

10. Chadwick A, Kaiser J, Vaccari C, et al. Online Social Endorsement and COVID-19 Vaccine Hesitancy in the United Kingdom. Social Media and Society 2021;7.

11. Puri N, Coomes EA, Haghbayan H, and Gunaratne K. Social media and vaccine hesitancy: new updates for the era of COVID-19 and globalized infectious diseases. Human Vaccines \& Immunotherapeutics 2020:1-8.

12. Murphy J, Vallières F, Bentall RP, et al. Psychological characteristics associated with COVID19 vaccine hesitancy and resistance in Ireland and the United Kingdom. Nature Communications 2021;12:1-15.

13. Calonico S, Cattaneo MD, and Titiunik R. Robust nonparametric confidence intervals for regression-discontinuity designs. Econometrica 2014;82:2295-326.

14. Cattaneo MD, Jansson M, and Ma X. Simple local polynomial density estimators. Journal of the American Statistical Association 2020;115:1449-55.

15. Larson HJ and Broniatowski DA. Volatility of vaccine confidence. Science 2021;371:12899.

16. Cattaneo MD, Jansson M, and Ma X. Local regression distribution estimators. Journal of Econometrics 2021. 


\section{Supplementary Files}

This is a list of supplementary files associated with this preprint. Click to download.

- Astrazenecavaccinationsluxembourg1017.pdf 